\title{
GLP1 and glucagon co-secreting pancreatic neuroendocrine tumor presenting as hypoglycemia after gastric bypass
}

\author{
Marta Guimarães, Pedro Rodrigues, Sofia S Pereira', Mário Nora, Gil Gonçalves, \\ Nicolai Wewer Albrechtsen², Bolette Hartmann², Jens Juul Holst ${ }^{2}$ and \\ Mariana P Monteiro ${ }^{1,3}$ \\ Department of General Surgery, Centro Hospitalar de Entre o Douro e Vouga, Rua Doutor Cândido Pinho, $4520-211$ \\ Santa Maria da Feira, Portugal \\ ${ }^{1}$ Clinical and Experimental Endocrinology, Department of Anatomy, Multidisciplinary Unit for Biomedical Research \\ (UMIB), Instituto de Ciências Biomédicas Abel Salazar, University of Porto (ICBAS/UP), Rua Jorge Viterbo Ferreira, \\ 228, 4050-313 Porto, Portugal \\ ${ }^{2}$ NNF Center for Basic Metabolic Research and Department of Biomedical Sciences, Faculty of Health and Medical \\ Sciences, University of Copenhagen, Copenhagen, Denmark \\ ${ }^{3}$ Endocrine Unit, Centro Hospitalar de Entre o Douro e Vouga, Rua Doutor Cândido Pinho, 4520-211 Santa Maria da \\ Feira, Portugal
}

Correspondence

should be addressed

to M P Monteiro

Email

mpmonteiro@icbas.up.pt

\section{Summary}

Post-prandial hypoglycemia is frequently found after bariatric surgery. Although rare, pancreatic neuroendocrine tumors (pNET), which occasionally are mixed hormone secreting, can lead to atypical clinical manifestations, including reactive hypoglycemia. Two years after gastric bypass surgery for the treatment of severe obesity, a 54-year-old female with previous type 2 diabetes, developed post-prandial sweating, fainting and hypoglycemic episodes, which eventually led to the finding by ultrasound of a $1.8-\mathrm{cm}$ solid mass in the pancreatic head. The $72-\mathrm{h}$ fast test and the plasma chromogranin A levels were normal but octreotide scintigraphy showed a single focus of abnormal radiotracer uptake at the site of the nodule. There were no other clinical signs of hormone secreting PNET and gastrointestinal hormone measurements were not performed. The patient underwent surgical enucleation with complete remission of the hypoglycemic episodes. Histopathology revealed a well-differentiated neuroendocrine carcinoma with low-grade malignancy with positive chromogranin A and glucagon immunostaining. An extract of the resected tumor contained a high concentration of glucagon (26.707 pmol/g tissue), in addition to traces of GLP1 $(471 \mathrm{pmol} / \mathrm{g})$, insulin $(139 \mathrm{pmol} / \mathrm{g})$ and somatostatin (23 pmol/g). This is the first report of a GLP1 and glucagon co-secreting PNET presenting as hypoglycemia after gastric bypass surgery. Although pNET are rare, they should be considered in the differential diagnosis of the clinical approach to the post-bariatric surgery hypoglycemia patient.

\section{Learning points:}

- pNETs can be multihormonal-secreting, leading to atypical clinical manifestations.

- Reactive hypoglycemic episodes are frequent after gastric bypass.

- pNETs should be considered in the differential diagnosis of hypoglycemia after bariatric surgery. 


\section{Background}

Post-prandial hypoglycemia is a rather frequent finding after bariatric surgery (1). Several mechanisms have been hypothesized as leading to hyperinsulinemic hypoglycemia following bariatric surgery, sharing in common a putative change in gut hormonal milieu (1). Hypoglycemia in patients who have undergone gastric bypass has been attributed to a number of mechanisms, including increased insulin sensitivity following weight loss, inappropriate insulin secretion after early entry of nutrients into the small intestine, lack of reduction of increased $\beta$-cell mass prior to surgery, surgically-induced changes in the secretion of insulinotropic incretin hormones and gut hormone activation of new $\beta$-cell formation with nesidioblastosis (2). While in very rare instances, the hypoglycemic episodes can result of endogenous hyperinsulinism due to previously unnoticed insulinoma (3).

The most frequent functioning pancreatic neuroendocrine tumor (pNET) are benign insulin secreting tumors leading to fasting hypoglycemia syndrome, followed by gastrin secreting tumors. pNETs secreting other peptides such as glucagon. Somatostatin, VIP and GLP1 are much less common (4) (5). pNET occasionally can be multihormonal with more than one peptidehormone-containing cell type and the hormone of the predominating cell type does not always explain the clinical symptoms presented by the patients (6).

\section{Case presentation}

A 54-year-old female previously diagnosed with type 2 diabetes was subjected to laparoscopic gastric bypass with a $200 \mathrm{~cm}$ biliopancreatic limb for treatment of morbid obesity (BMI $42 \mathrm{~kg} / \mathrm{m}^{2}$ ). Two years after bariatric surgery the patient developed post-prandial episodes of sweating and fainting associated with documented hypoglycemia (capillary blood glucose $<50 \mathrm{mg} / \mathrm{dl}$ ) in spite of appropriate nutrition. At that point, the patient was not actively losing weight with a stabilized BMI of $30 \mathrm{~kg} / \mathrm{m}^{2}$ and type 2 diabetes was in clinical remission as of the 6th postoperative month.

\section{Investigation}

Abdominal ultrasound revealed a $1.8-\mathrm{cm}$ solid mass in the pancreatic head that was confirmed by abdominal CT scan (Fig. 1A). Octreotide scan showed a focus of abnormal uptake of the radiotracer in the topography of the pancreatic lesion without other foci of abnormal

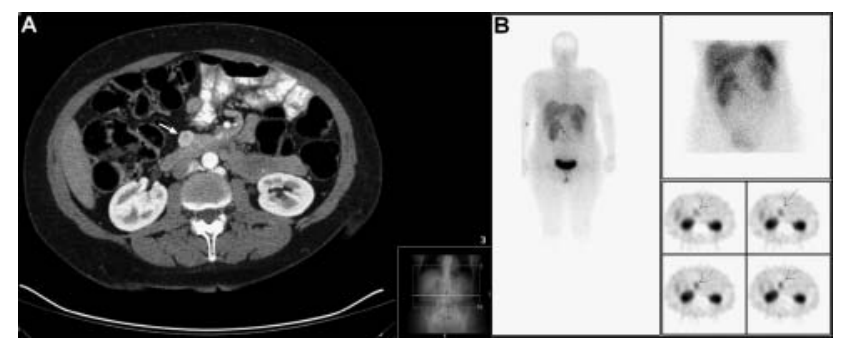

Figure 1

Abdominal CT scan revealing a 1.8- $\mathrm{cm}$ solid mass in the pancreatic head (white arrow) (A); octreotide scan showing a focus of abnormal uptake of the radiotracer in the same topography of the pancreatic lesion without other foci of abnormal uptake of the radiotracer (B).

uptake (Fig. 1B). The lowest laboratory glucose and parallel insulin recorded were $65 \mathrm{mg} / \mathrm{dl}(3.6 \mathrm{mmol} / \mathrm{l})$ and $3.3 \mu \mathrm{UI} / \mathrm{ml}$ respectively. Plasma chromogranin A levels were $4.1 \mathrm{ng} / \mathrm{ml}$ (normal $<6 \mathrm{ng} / \mathrm{ml}$ ) and the 72 -h fast test was negative for hypoglycemia.

\section{Treatment}

The patient underwent laparoscopic enucleation of the pancreatic nodule for suspected pNET. A small pancreatic nodule was found at the upper edge of the head of the pancreas. Intraoperative pancreatic ultrasound revealed that the pancreatic nodule was more than $1 \mathrm{~cm}$ away from the pancreatic duct and there was no evidence of other nodules. The postoperative period was uneventful, with complete remission of the hypoglycemic episodes ever since.

\section{Outcome and follow-up}

The histology of the surgical specimen showed a $18 \times 15 \times$ $14 \mathrm{~mm}$ pseudoglandular neuroendocrine neoplasia staining positive for CAM 5.2, chromogranin A, synaptophysin, glucagon and GLP1, and negative for insulin, with a low Ki-67 $(<2 \%)$ and mitotic index ( $<1$ mitosis/10c). Such findings were indicative of a low-grade (G1) well-differentiated neuroendocrine tumor (Fig. 2).

PET-68aGa-SRP (DOTANOC), performed postoperatively, excluded the existence of any residual disease.

Frozen tumor sample was homogenized in $1 \%(\mathrm{v} / \mathrm{v})$ trifluoroacetic acid, extracts were purified and eluted with $70 \%$ ethanol. After overnight drying were reconstituted in TRIS buffer supplemented with $0.1 \%(\mathrm{w} / \mathrm{v})$ human serum albumin, EDTA and thimerosal (7). Peptide hormone levels were measured by in-house developed RIAs with an approximate sensitivity of $1 \mathrm{pmol} / \mathrm{l}$ and intra-assay 

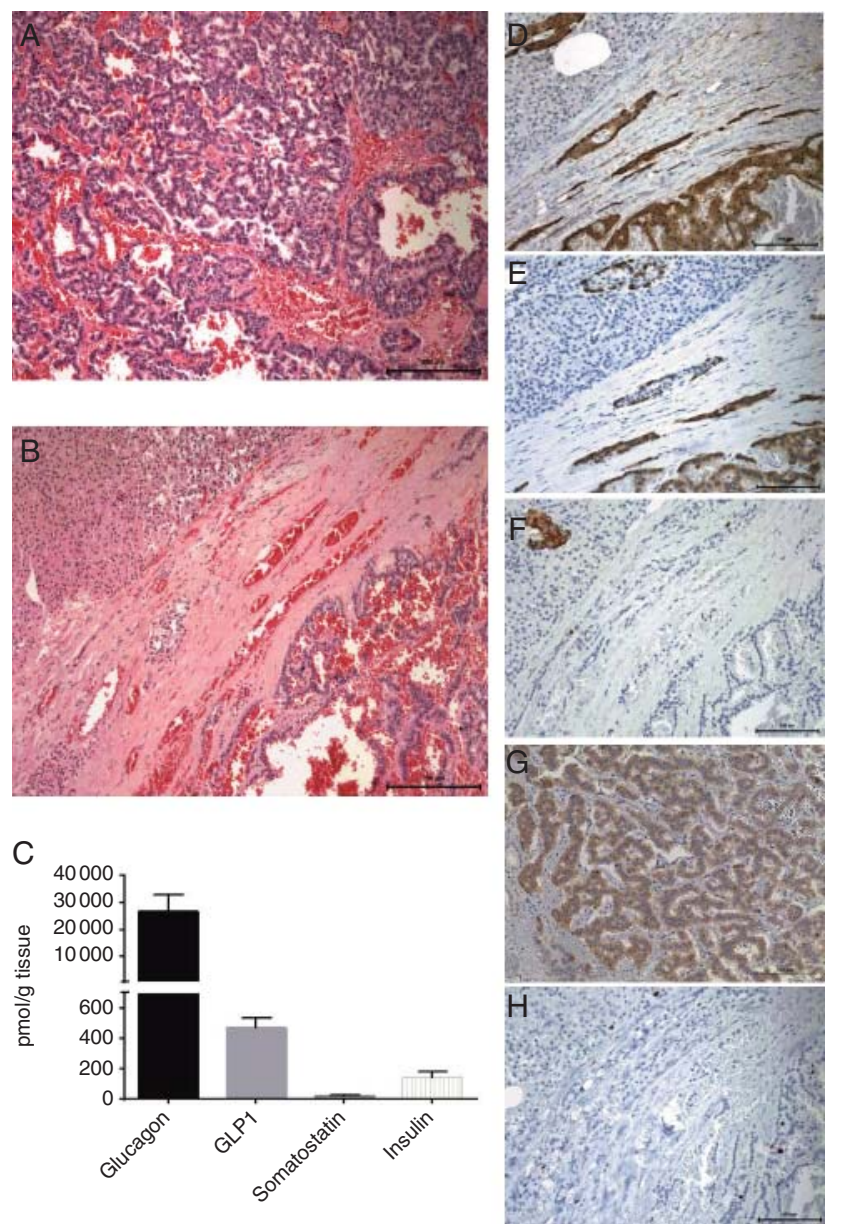

Figure 2

Photographs of histological findings of the hematoxylin eosin staining of the pNET $(100 \times)(A)$ and surrounding normal pancreatic tissue on the top left corner $(100 \times)(B)$; graphic illustration of tumor extract tissue concentrations (pmol/g tissue) of glucagon, GLP1, somatostatin and insulin (mean \pm s.D. of technical replicates) (C); immunostaining of the glucagonoma for the neuroendocrine markers chromogranin $A(200 \times)$ (D), glucagon $(200 \times)(E), G L P 1(F)$ and insulin $(200 \times)(G)$; the proliferation index displayed by the low Ki-67 staining $(200 \times)(\mathrm{H})$ (immune staining in brown).

coefficient of variation $<6 \%$. The tumor showed a high concentration of glucagon $(26.707 \mathrm{pmol} / \mathrm{g}$ tissue) and traces of GLP1 (471 pmol/g), insulin (139 pmol/g) and somatostatin (23 pmol/g) (Fig. 2).

\section{Discussion}

Gastric bypass surgery has been reported to have an important antidiabetic effect that has been hypothesized to be due to increased GLP1 secretion, which in turn stimulates insulin (2). $\alpha$-cell function has also been reported to change after gastric bypass and to show increased glucagon release after meals (8). The distinctive prandial insulin and glucagon responses after meals in subjects previously subjected to gastric bypass suggest that regulatory influences on islet function may be dramatically altered by gastric bypass (8). The endocrine and metabolic alterations, induced not only by the weight loss but also by the anatomical rearrangement of the gastrointestinal tract, have resulted in an unexpected consequence of bariatric surgery and the frequent occurrence of post-prandial hypoglycemic episodes. While in rare instances, the hypoglycemic episodes can be a consequence of endogenous hyperinsulinism due to previously unnoticed insulin secreting or de novo pNET (3) (9). Therefore, although uncommon, the possibility of a functioning pNET should be considered in clinical approach to post-bariatric surgery hypoglycemia.

The most frequent functioning pNET are benign insulin secreting tumors leading to fasting hypoglycemia syndrome. The differential diagnosis between noninsulinoma pancreatogenous hypoglycemia and insulinoma can usually be made since the post-prandial symptomatology is predominant and hypoglycemia occurs after the oral glucose tolerance test but not after a 72 -h fast in the noninsulinoma hypoglycemia. However, up to one third of pNET can secrete several peptide hormones, resulting in a variety of rather unique clinical syndromes, although expression of three different hormones by the same tumor was only found to be present in $1.3 \%$ of the cases (10). In addition, in mixed tumors, the predominant cell type hormone sometimes does not explain the clinical symptoms of the patient who may present with symptoms characteristic of hypersecretion of only one of the other hormones (5). Furthermore, conventional staining methods are not always able to distinguish between the different hormone-producing cell types, rendering the diagnosis even more challenging (5).

In this case, given that the tumor was a small nodule in the head of the pancreas without associated glucagonoma syndrome, preoperative diagnosis of glucagonoma was not considered and the diagnosis of GLP1/glucagon co-secreting pNET was made by GLP1 and glucagon immunostaining and by demonstrating the tumor's high content of glucagon. In spite of the documented hypoglycemic episodes, which led to the incidental finding of the neuroendocrine tumor, there was no biochemical confirmation of hyperinsulinemic hypoglycemia. Although the tumor was found to contain insulin, it is highly unlikely that this was responsible for the hypoglycemic episodes since the $72 \mathrm{~h}$ fast was negative and the hypoglycemia 
was post-prandial. In addition, the tumor was also found to contain GLP1, derived from the same preproglucagon gene product (11). The secretion of GLP1 by a pNET has previously been described in a patient with pNET (12), and could have contributed to the observed reactive hypoglycemic episodes, possibly in addition to the increase in GLP1 induced by the surgery. However, the direct secretion of GLP1 by the tumor becomes more likely given the resolution of hypoglycemia after surgery. The result of the tumor peptide content analysis, revealing the presence of glucagon, GLP1 and insulin, further supports the histopathology diagnosis of a well-differentiated pNET. If glucagon was excessively secreted in the blood, another possible explanation for the patient's hypoglycemic episodes could have been the aggravated post-prandial glucose excursion with subsequent incretinmediated insulin secretion followed by reactive hypoglycemia (13).

The influence of these hormonal alterations in the risk of development of pancreatic neoplasia is a question of debate that deserves to be investigated. Pancreatic pathology of patients subjected to gastric bypass revealed islet cell hyperplasia and expansion of the $\beta$-cell mass (2). Also, $\alpha$-cell hyperplasia has been found after incretin therapy (14), although that report was heavily criticized. Nevertheless, these alterations may represent an extreme of the metabolic benefit and altered physiology that accompanies gastric bypass surgery.

\section{Conclusion}

This is the first report of a GLP1/glucagon co-secreting pNET presenting as hypoglycemia after bypass surgery. pNETs, although rare, should be considered in the differential diagnosis of the post-bariatric patient presenting with hypoglycemic episodes. The current GLP1/ glucagon co-secreting tumor presenting after bariatric might have been coincidental, however, it raises once again the question whether the metabolic changes surgically induced by the anatomical rearrangements could lead to cellular hyperplasia and neoplasia.

\section{Declaration of interest}

The authors declare that there is no conflict of interest that could be perceived as prejudicing the impartiality of the research reported.

\section{Funding}

Unit for Multidisciplinary Research in Biomedicine is funded by grants from the Foundation for Science and Technology (PEst-OE/SAU/UI0215/2014;
Fcomp-01-0124-FEDER-015896). S S Pereira holds a PhD grant from the Foundation for Science and Technology (SFRH/BD/89308/2012).

\section{Patient consent}

Written informed consent has been obtained from the patient for publication of the submitted article and accompanying images.

\section{Author contribution statement}

M Guimarães wrote the manuscript and was responsible for the patient assessment and treatment; P Rodrigues, M Nora, G Gonçalves assessed and treated the patient; S S Pereira, N W Albrechtsen, B Hartmann, J J Holst were responsible for the investigation of histological samples and peptide characterization, M P Monteiro assessed the patient and reviewed the manuscript.

\section{Acknowledgements}

The authors would like to acknowledge Prof. Carlos Lopes for his support in histopathology classification.

\section{References}

1 Kefurt R, Langer FB, Schindler K, Shakeri-Leidenmuhler S, Ludvik B \& Prager G 2015 Hypoglycemia after Roux-En-Y gastric bypass: detection rates of continuous glucose monitoring (CGM) versus mixed meal test. Surgery for Obesity and Related Diseases 11 564-569. (doi:10.1016/j.soard. 2014.11.003)

2 Dirksen C, Jorgensen NB, Bojsen-Moller KN, Jacobsen SH, Hansen DL, Worm D, Holst JJ \& Madsbad S 2012 Mechanisms of improved glycaemic control after Roux-en-Y gastric bypass. Diabetologia $\mathbf{5 5}$ 1890-1901. (doi:10.1007/s00125-012-2556-7)

3 Zagury L, Moreira RO, Guedes EP, Coutinho WF \& Appolinario JC 2004 Insulinoma misdiagnosed as dumping syndrome after bariatric surgery. Obesity Surgery 14 120-123. (doi:10.1381/096089204772787419)

4 Halfdanarson TR, Rubin J, Farnell MB, Grant CS \& Petersen GM 2008 Pancreatic endocrine neoplasms: epidemiology and prognosis of pancreatic endocrine tumors. Endocrine-Related Cancer 15 409-427. (doi:10.1677/ERC-07-0221)

5 Vinik AI, Woltering EA, Warner RR, Caplin M, O'Dorisio TM, Wiseman GA, Coppola D \& Go VL 2010 NANETS consensus guidelines for the diagnosis of neuroendocrine tumor. Pancreas 39 713-734. (doi:10.1097/MPA.0b013e3181ebaffd)

6 Larsson LI, Grimelius L, Hakanson R, Rehfeld JF, Stadil F, Holst J, Angervall L \& Sundler F 1975 Mixed endocrine pancreatic tumors producing several peptide hormones. American Journal of Pathology $\mathbf{7 9}$ 271-284.

7 Kuhre RE, Albrechtsen NW, Windelov JA, Svendsen B, Hartmann B \& Holst JJ 2014 GLP-1 amidation efficiency along the length of the intestine in mice, rats and pigs and in GLP-1 secreting cell lines. Peptides 55 52-57. (doi:10.1016/j.peptides.2014.01.020)

8 Goldfine AB, Mun EC, Devine E, Bernier R, Baz-Hecht M, Jones DB, Schneider BE, Holst JJ \& Patti ME 2007 Patients with neuroglycopenia after gastric bypass surgery have exaggerated incretin and insulin secretory responses to a mixed meal. Journal of Clinical Endocrinology and Metabolism 92 4678-4685. (doi:10.1210/jc.2007-0918)

9 Foster-Schubert KE 2011 Hypoglycemia complicating bariatric surgery: incidence and mechanisms. Current Opinion in Endocrinology, Diabetes, and Obesity 18 129-133. (doi:10.1097/MED.0b013e32834449b9)

10 Kim JY, Kim MS, Kim KS, Song KB, Lee SH, Hwang DW, Kim KP, Kim HJ, Yu E, Kim SC et al. 2015 Clinicopathologic and prognostic significance 
Endocrinology,

Diabetes \& Metabolism

CASE REPORTS
M Guimarães and others

GLP1 and glucagon co-secreting pNET
ID: 15-0049; August 2015

DOI: 10.1530/EDM-15-0049 of multiple hormone expression in pancreatic neuroendocrine tumors. American Journal of Surgical Pathology 39 592-601. (doi:10.1097/ PAS.0000000000000383)

11 Ugleholdt R, Zhu X, Deacon CF, Orskov C, Steiner DF \& Holst JJ 2004 Impaired intestinal proglucagon processing in mice lacking prohormone convertase 1. Endocrinology 145 1349-1355. (doi:10.1210/ en.2003-0801)

12 Roberts RE, Zhao M, Whitelaw BC, Ramage J, Diaz-Cano S, le Roux CW, Quaglia A, Huang GC \& Aylwin SJ 2012 GLP-1 and glucagon secretion from a pancreatic neuroendocrine tumor causing diabetes and hyperinsulinemic hypoglycemia. Journal of Clinical Endocrinology and Metabolism 97 3039-3045. (doi:10.1210/jc.2011-2005)

13 Halperin F, Patti ME \& Goldfine AB 2010 Glucagon treatment for post-gastric bypass hypoglycemia. Obesity 18 1858-1860. (doi:10.1038/ oby.2010.15)

14 Butler AE, Campbell-Thompson M, Gurlo T, Dawson DW, Atkinson M $\&$ Butler PC 2013 Marked expansion of exocrine and endocrine pancreas with incretin therapy in humans with increased exocrine pancreas dysplasia and the potential for glucagon-producing neuroendocrine tumors. Diabetes 62 2595-2604. (doi:10.2337/db12-1686)

Received in final form 7 July 2015

Accepted 15 July 2015 